\title{
Ik6 expression provides a new strategy for the therapy of acute lymphoblastic leukemia
}

\author{
FEN ZHOU, YUNYUN XU, YINING QIU, XIAOYAN WU, ZHIQUAN ZHANG and RUNMING JIN \\ Department of Pediatrics, Union Hospital, Tongji Medical College, Huazhong University \\ of Science and Technology, Wuhan, Hubei 430022, P.R. China
}

Received November 13, 2013; Accepted December 16, 2013

DOI: $10.3892 /$ or.2014.2969

\begin{abstract}
Our previous study demonstrated that the dominantnegative Ikaros isoform 6 (Ik6) is overexpressed in Chinese children with newly diagnosed B-acute lymphoblastic leukemia (B-ALL) and is strongly associated with a poor outcome. The purpose of the present study was to further explore the function of Ik6 in B-ALL. The association between Ik6 expression as detected by real-time PCR and efficacy of chemotherapy was evaluated. The effect of the alteration in Ik6 on leukemic cell lines was assessed by in vitro gain-of-function and loss-offunction techniques. PCR analysis showed that Ik6 expression was decreased when patients completed induction chemotherapy and reached complete remission. Ik6 expression was significantly increased when patients suffered relapse. Stable transfection of Ik6 into the Nalm-6 cell line revealed that Ik6 enhanced proliferation of Nalm- 6 cells through the promotion of G0/G1-to-S-phase transition and enhanced chemoresistance to chemotherapeutics through anti-apoptotic effects. However, Ik6 expression did not affect the invasion of Nalm-6 cells. In contrast, silencing of Ik6 in Sup-B15 cells significantly inhibited proliferation and increased chemosensitivity. The present study suggests that Ik6 may be a biological marker of chemosensitivity and relapse and Ik6 may provide a potential therapeutic strategy for ALL.
\end{abstract}

\section{Introduction}

In spite of continuous progress in the therapy of acute lymphoblastic leukemia (ALL), relapses still occur in up to $20 \%$ of children and most adults with ALL (1-4). Moreover, the outcome of relapsed ALL patients is extremely poor $(3,5,6)$. To improve the survival of ALL patients, it is critical to identify new molecular biomarkers such as BCR/ABL which are

Correspondence to: Professor Runming Jin, Department of Pediatrics, Union Hospital, Tongji Medical College, No. 1277 Jiefang Road, Wuhan, Hubei 430022, P.R. China

E-mail: jinrunming@126.com

Key words: Ikaros isoform 6, acute lymphoblastic leukemia, malignant biological characteristics involved in the regulation of the malignant biological behavior of leukemia cells and are valuable in the prognosis and therapy of leukemia patients.

Ikaros is a lymphoid transcription factor that was identified as a hematological tumor suppressor $(7,8)$. Only Ikaros isoforms that contain at least three DNA-binding zinc fingers possess functional activity, such as Ik1, Ik2 and Ik3. Isoforms lacking two or more zinc-finger domains cannot bind DNA and impair the function of Ikaros proteins in a dominant-negative manner (9). Dominant-negative Ikaros isoform 6, (Ik6), with a deletion of coding exons 3 through 6 , is the most common and strongest transcriptional repressor in the Ikaros family (10-12). Ample evidence indicates that overexpression of Ik6 is associated with a poor prognosis of ALL patients (13-15). A recent study showed that the prognosis of Ph-negative patients with Ik6 was close to that of Ph-positive patients (16).

The clinical data available to date suggest that Ik6 should be evaluated as a prognostic marker for newly diagnosed ALL patients and may be involved in leukemogenesis. However, there are few studies concerning the role of Ik6 in the therapy of ALL. In the present study, in order to ascertain whether Ik6 is a marker of chemotherapeutic efficacy and is a potential therapeutic target, we investigated changes in Ik6 expression during treatment of ALL patients and assessed the effects in vitro of Ik6 expression on cell proliferation, cell cycle, chemosensitivity to vincristine (VCR), daunorubicin (DNR) and L-asparaginase (L-Asp) and invasion in ALL cell lines.

\section{Materials and methods}

Patients and samples. The 25 patients included in the present study were diagnosed with Ik6-positive B lineage ALL between January 2009 and January 2012 and were treated at Wuhan Union Hospital, in accordance with the CCLG-ALL-2008 Protocol (Children's Cancer and Leukemia Group). All samples were bone marrow aspirates and were obtained following informed consent in strict accordance with the Declaration of Helsinki. The endpoint of the present study was January 2013, and the expression of Ik6 was measured at the point of initial diagnosis, end of induction therapy, at complete remission and at relapse.

Cell culture. Sup-B15 and Nalm-6, human B-cell precursor leukemia cell lines, were used for the study. Both cell lines 
were purchased from the American Type Culture Collection (ATCC) (Rockville, MD, USA) and cultured in RPMI-1640 medium supplemented with $10 \%$ fetal bovine serum (FBS) (both from Gibco-BRL, Carlsbad, CA, USA) at $37^{\circ} \mathrm{C}$ in a humidified atmosphere with $5 \% \mathrm{CO}_{2}$.

Overexpression or silencing of Ik6 in acute lymphoblastic leukemia cell lines. Nalm-6 cells stably overexpressing Ik6 were obtained as follows. The complete Ik6 coding sequence was amplified by PCR from Sup-B15 cells and cloned into the lentiviral expression vector pHR-SIN-CSIGW. The vector pHR-CSIGW-Ik6 and the viral packaging system (containing an optimized mixture of two packaging plasmids, pMD2.G and psPAX2) were co-transfected into $293 \mathrm{~T}$ cells to produce competent lentivirus. The viral supernatant was harvested at $48 \mathrm{~h}$ post-transfection and was used to infect Nalm-6 cells. The pHR-CSIGW-mock vector was also packaged and used as a negative control. For transfection, $1 \times 10^{6} \mathrm{Nalm}-6$ cells were collected on day 2 and resuspended in $1 \mathrm{ml}$ complete medium (RPMI-1640 medium supplemented with 10\% FBS). Cells were transfected with pHR-CSIGW-Ik6 or pHR-CSIGW-mock at a multiplicity of infection (MOI) of 50 for $8 \mathrm{~h}$. Then half of the above medium was replaced with $1 \mathrm{ml}$ fresh medium. Thereafter, cells were cultured for another $64 \mathrm{~h}$ and analyzed for expression of GFP by flow cytometry. The expression of Ik6 protein was further confirmed by western blotting.

Sup-B15 cells with stably silenced Ik6 were obtained through a similar procedure. Firstly, we designed several small interfering RNAs (siRNAs) and screened the most effective one. The target sequence for Ik6 was 5'-GCTACGAGAAGG AGAACGA-3' and the negative control sequence was 5'-TTC TCCGAACTGTCACGT-3'. Then, the small hairpin RNA (shRNA) was cloned into the self-inactivating lentiviral vector (GeneChem, Shanghai, China) containing a CMV-driven GFP reporter and a $\mathrm{U} 6$ promoter upstream of the cloning sites (AgeI and EcoRI).

Real-time RT-PCR assay. Total cellular RNA was extracted from cells using TRIzol reagent and converted to singlestranded cDNA using the Toyobo kit. Real-time PCR amplification was performed using the SYBR-Green Master Mix (Toyobo, Japan) and the StepOnePlus ${ }^{\text {TM }}$ Real-Time PCR System (Bio-Rad, Hercules, CA, USA). The primers for Ik6, vascular endothelial growth factor (VEGF), vascular endothelial growth factor receptor (Flt-1), placenta growth factor fragment (PlGF), angiogenin-1 (Ang-1), angiogenin-2 (Ang-2), matrix metalloproteinase-2 (MMP-2) and matrix metalloproteinase-9 (MMP-9) are shown in Table I. Cycling conditions were $95^{\circ} \mathrm{C}$ for $30 \mathrm{sec}$ followed by 40 cycles of $95^{\circ} \mathrm{C}$ for $10 \mathrm{sec}$ and $60^{\circ} \mathrm{C}$ for $35 \mathrm{sec}$. The relative levels of mRNA expression were quantified by comparison with the internal control (GAPDH). All the samples were performed in triplicate, and the results were analyzed using the $2^{-\Delta \Delta C t}$ method.

Western blotting. Cells were lysed using a nuclear and cytoplasmic protein extraction kit (Beyotime, China) according to the manufacturer's instructions. The extracts were centrifuged at $12,000 \mathrm{rpm}$ for $15 \mathrm{~min}$ at $4^{\circ} \mathrm{C}$, and the supernatant was collected. A BCA protein assay kit (Pierce, Rockford, IL, USA) was used to determine the protein concentrations. Samples
Table I. Sequences of primers used in real-time PCR.

\begin{tabular}{lll}
\hline Gene & & Primer sequences \\
\hline Ik6 & F & ccctgtaagcgatactccag \\
& R & ttgtccccacgact \\
VEGF & F & atcttcaagccatcctgtgtgc \\
& R & gctcaccgcctcggcttgt \\
Flt-1 & F & atcattccgaagcaaggtgtg \\
& R & aaacccattggcacatctgt \\
PlGF & F & cacttcccctgttcttctgaa \\
& R & caagcaaatggcaagtgtga \\
Ang-1 & F & ttcctttctttgcttcctc \\
& R & ctgcagagcgtttgtgttgt \\
Ang-2 & F & aacatcccagtccacctgag \\
& R & ggtcttgcttggtccgtta \\
MMP-2 & F & ggaagcatcaatcggactg \\
& R & gggcgggagaaagtagca \\
MMP-9 & F & cccacttactttggaaacg \\
& R & gaagatgaatggaaatacgc \\
GAPDH & F & cctccaaggagtaagaccc \\
& R & aggggtctacatggcaactg \\
\hline
\end{tabular}

F, forward; R, reverse; Ik6, Ikaros isoform 6; VEGF, vascular endothelial growth factor; Flt-1, vascular endothelial growth factor receptor; PIGF, placenta growth factor fragment; Ang-1, angiogenin-1; Ang-2, angiogenin-2; MMP-2, matrix metalloproteinase-2; MMP-9, matrix metalloproteinase-9.

were resolved in 10\% SDS-PAGE and then transferred onto nitrocellulose membranes (Bio-Rad). After being blocked with $5 \%$ skim milk in Tris-buffered saline with $0.1 \%$ Tween-20, proteins were detected by respective antibodies using an ECL kit (Pierce) and exposed to X-ray film. Blots were visualized with a western blotting detection system (Bio-Rad).

Proliferation assay. To investigate the effects of the alteration in expression Ik6 on leukemia cells, exponentially growing cells $\left(2 \times 10^{4} /\right.$ well $)$ were seeded in quintuplicate in 96-well plates. After seeding for 1, 2, 3 and 4 days, the quantity of viable cells was determined. Ten microliters of CCK-8 Cell Counting Reagent (Dojindo Molecular Technologies, Inc., Japan) was added directly to each well. The plates were sequentially incubated for $4 \mathrm{~h}$ at $37^{\circ} \mathrm{C}$, and the WST- 8 formazan product was measured at $490 \mathrm{~nm}$ using a microplate reader (Tecan Sunrise, Switzerland). To investigate the effects of the alteration in Ik6 expression combined with chemotherapeutics on leukemia cells, cells were incubated with culture medium containing various concentrations of the chemotherapeutics [2.5 to $50 \mathrm{ng} / \mathrm{ml}$ for vincristine (VCR); 2.5 to $50 \mathrm{ng} / \mathrm{ml}$ for daunorubicin (DNR) and 0.1 to $2.5 \mathrm{IU} / \mathrm{ml}$ for L-asparaginase (L-Asp)], respectively. After allowing cells to grow for $24 \mathrm{~h}$ at $37^{\circ} \mathrm{C}$, the viable cell population in each well was reflected by the OD values. Then the fraction of surviving cells was calculated and the $\mathrm{IC}_{50}$ was determined by nonlinear regression analysis using SPSS 11.5 software. 
A

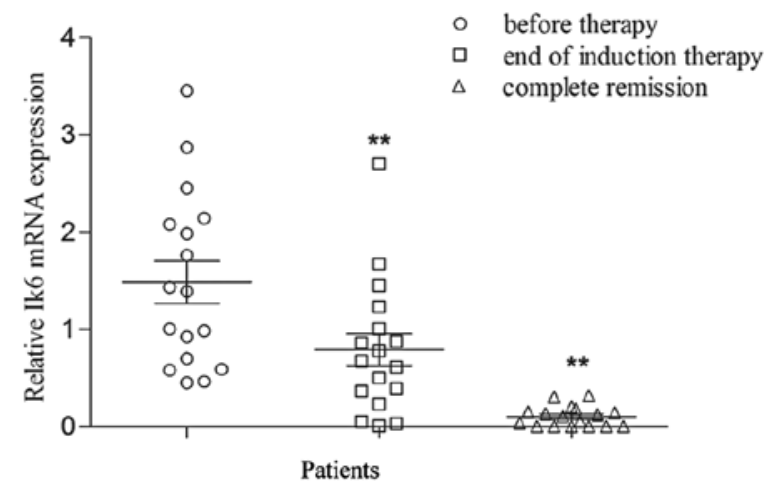

B

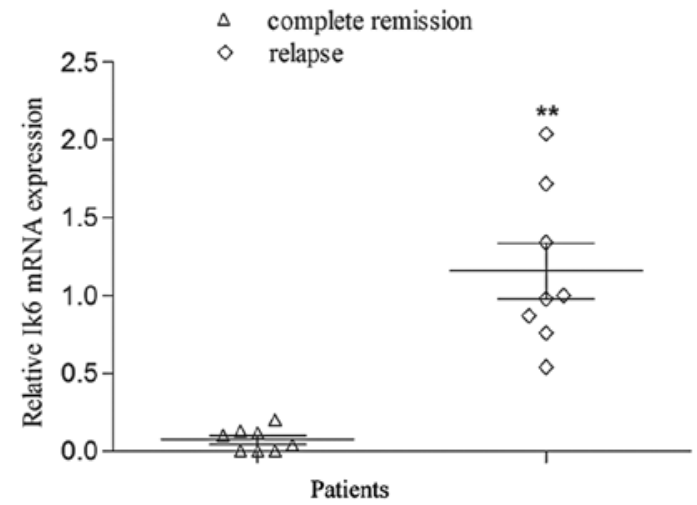

Figure 1. Ik6 expression of 25 B-ALL children during chemotherapy. (A) Ik6 expression was decreased when patients completed induction chemotherapy $\left({ }^{* *} \mathrm{P}<0.01, \mathrm{n}=17\right)$, or reached complete remission $\left({ }^{* *} \mathrm{P}<0.01, \mathrm{n}=17\right)$. (B) In contrast, Ik6 expression was increased when patients suffered relapse $\left({ }^{* *} \mathrm{P}<0.01\right.$, $\mathrm{n}=8$ ). Ik6, Ikaros isoform 6; B-ALL, B-acute lymphoblastic leukemia.

Cell cycle analysis. The cells $\left(10^{6}\right.$ cells) were collected and fixed with ice-cold $70 \%$ ethanol overnight at $4^{\circ} \mathrm{C}$. After washing with PBS and resuspension, fixed cells were treated with $50 \mu \mathrm{g} / \mathrm{ml}$ RNase A (Amresco Inc., Solon, OH, USA) for $15 \mathrm{~min}$ at $37^{\circ} \mathrm{C}$, and then incubated with $5 \mu \mathrm{g} / \mathrm{ml}$ propidium iodide (Sigma Chemical Co., St. Louis, MO, USA) for $30 \mathrm{~min}$ at room temperature in the dark. The cell cycle distribution was detected by flow cytometry (Becton-Dickinson, Franklin Lakes, NJ, USA).

Analysis of apoptosis. The apoptosis detection kit was from KeyGen Biotech (Nanjing, China). Cells were incubated with culture medium containing final concentrations of $5.0 \mathrm{ng} / \mathrm{ml}$ VCR, $0.5 \mathrm{ng} / \mathrm{ml} \mathrm{DNR}$ and $0.1 \mathrm{IU} / \mathrm{ml} \mathrm{L}-\mathrm{Asp}$, respectively, for $24 \mathrm{~h}$. Treated cells were stained with propidium iodide and Annexin V-FITC for $15 \mathrm{~min}$ according to the manufacturer's instructions. The stained cells were subjected to flow cytometric analysis.

Cell migration and invasion assays. Cell migration was evaluated using an uncoated Transwell assay. Cells $\left(2 \times 10^{5}\right)$ were suspended in $200 \mu \mathrm{l}$ of serum-free RPMI-1640 medium and placed in the upper chambers of the Transwell plate (Corning, Cambridge, MA, USA). RPMI medium plus 10\% FBS (250 $\mu \mathrm{l})$ and NIH3T3-conditioned medium $(250 \mu \mathrm{l})$ were added to the lower chambers. Plates were incubated at $37^{\circ} \mathrm{C}$ for $8 \mathrm{~h}$. The cells of the lower compartments were counted, and the rate of
A
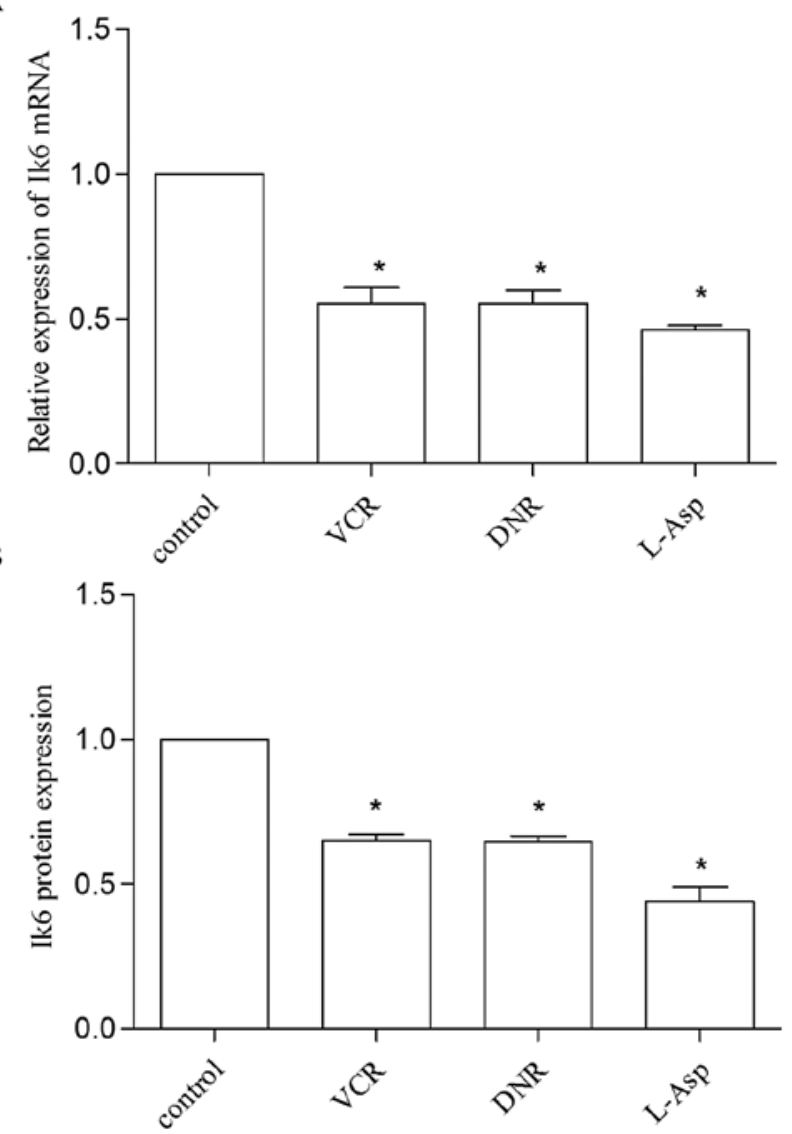

Ik6

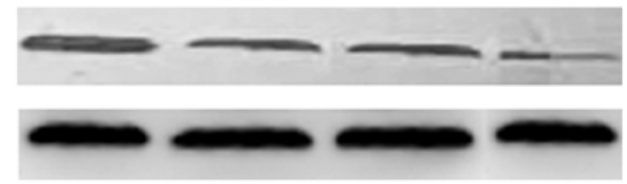

Figure 2. Effect of chemotherapeutics on the expression of Ik6 in Sup-15 cells. Treatment with VCR, DNR and L-Asp significantly decreased the (A) mRNA and (B) protein expression of Ik6 ( $\mathrm{P}<0.05, \mathrm{n}=3)$ in Sup-15 cells. Ik6, Ikaros isoform 6; VCR, vincristine; DNR, daunorubicin; L-Asp, L-asparaginase.

migration was expressed as a percentage of the total number of cells added to each well. The cell invasion assay was similar to the migration assay but a Matrigel-coated Transwell was used.

Statistical analysis. Data are presented as means \pm SD. Comparisons between groups were carried out by the Student's t-test with software SPSS 11.5. Differences were considered to be statistical significant at $\mathrm{P}<0.05$.

\section{Results}

Expression of Ik6 during chemotherapy. In the 25 patients with Ik6-positive expression, 8 responded poorly to chemotherapy. Seventeen patients achieved complete remission but 8 patients of these 17 patients later suffered from relapse. After induction chemotherapy, the Ik6 expression was significantly downregulated compared with that before treatment $(\mathrm{P}<0.01)$. However, in the children with relapse, Ik6 expression was again increased $(\mathrm{P}<0.01)$ (Fig. 1). We also determined the alteration 
A

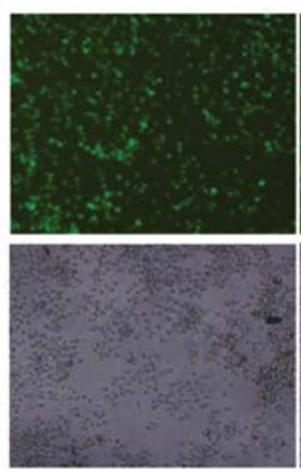

control
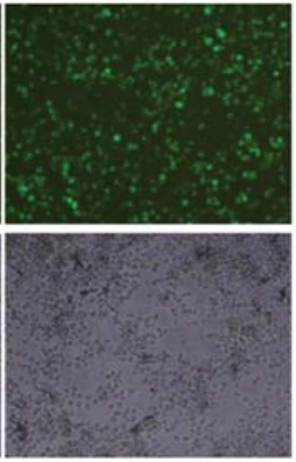

Ik6
B

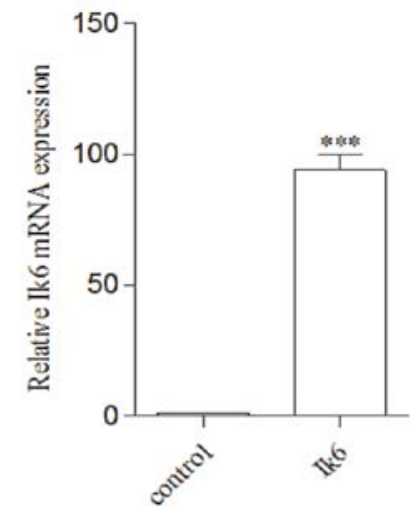

Ik6

$\beta$-actin

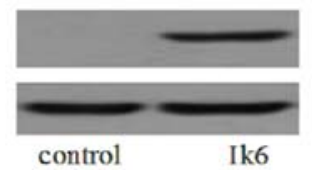

Figure 3. Lentiviral-mediated expression of Ik6 in Nalm-6 cells. (A) Expression of GFP in Nalm-6 cells transfected with Ik6 or control for $72 \mathrm{~h}$. (B and C) The expression levels of mRNA and protein of Ik6 were confirmed by real-time RT-PCR and western blotting $\left.{ }^{(* * *} \mathrm{P}<0.001, \mathrm{n}=3\right)$. Ik6, Ikaros isoform 6 .

A

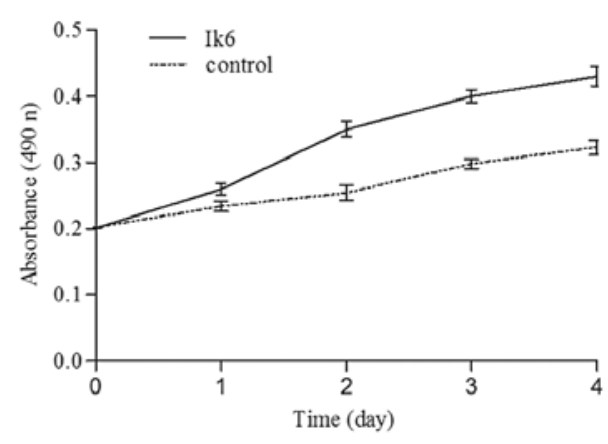

B

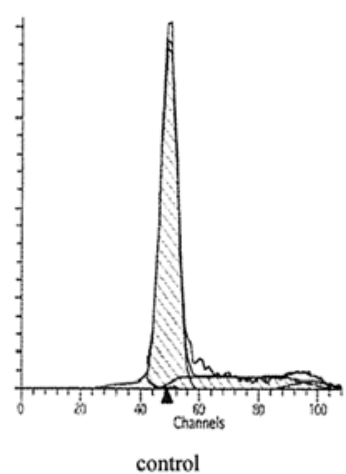

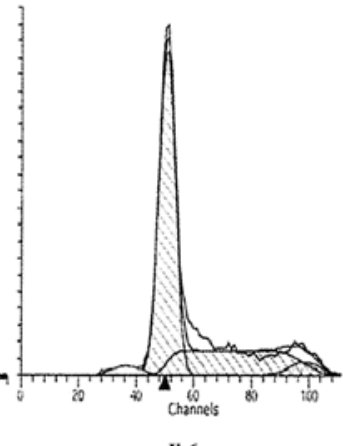

Ik6

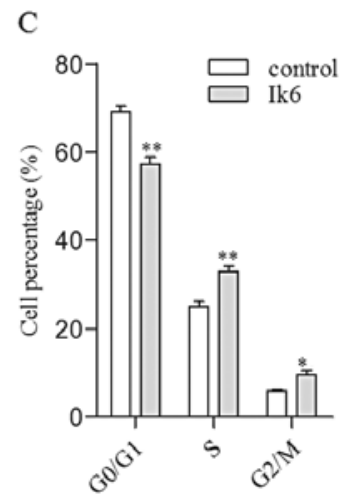

Figure 4. Ik6 overexpression promotes the proliferation of Nalm- 6 cells. (A) The CCK-8 assay indicated that the percentage of viable cells in the Nalm-6/Ik6 group was significantly higher than those in the control group during the culture period $(\mathrm{P}<0.05, \mathrm{n}=5)$. (B and $\mathrm{C})$ The result of the cell cycle analysis showed that Nalm-6/Ik6 cells exhibited a decreased proportion of cells in the static phase (G0/G1) and an increased proportion in the synthesis (S) and mitotic phase $(\mathrm{G} 2 / \mathrm{M})$ of the cell cycle. $\left({ }^{*} \mathrm{P}<0.05,{ }^{* *} \mathrm{P}<0.01, \mathrm{n}=3\right)$. Ik6, Ikaros isoform 6.

in Ik6 expression in the Sup-B15 cell line after co-culture with different chemotherapeutics. A similar result was found in that VCR, DNR and L-Asp treatment markedly decreased the Ik6 mRNA and protein expression $(\mathrm{P}<0.05)$ (Fig. 2).

Overexpression of Ik6 in Nalm-6 cells enhances cell proliferation and alters cell cycle distribution. Nalm-6 cells expressed GFP after being transfected with Ik6 or with the control, and GFP fluorescence was observed in almost $95 \%$ of the cells (Fig. 3A). Real-time PCR and immunoblotting showed a generally higher level of Ik6 mRNA and protein expression in Nalm-6 cells following Ik6 overexpression (Fig. 3B and C).

After transfection with Ik6, Nalm-6/Ik6 cells exhibited increased cell proliferation compared with the Nalm-6 cells lacking the protein (Fig. 4A, $\mathrm{P}<0.05, \mathrm{n}=5$ ). Cell cycle results showed that Ik6-expressing Nalm- 6 cells exhibited a decreased proportion of cells in the static phase (G0/G1) and an increased proportion in the synthetic (S) and mitotic phases (G2/M) of the cell cycle (Fig. 4B and C). Therefore, expression of Ik6 in Nalm-6 cells promoted cell cycle progression from the $\mathrm{G} 0 / \mathrm{G} 1$ phase to the $\mathrm{S}$ and $\mathrm{G} 2 / \mathrm{M}$ phase.

Overexpression of Ik6 in Nalm-6 cells decreases sensitivity to chemotherapeutics through an anti-apoptotic effect. The effects of VCR, DNR and L-Asp on the growth of leukemia cells were evaluated, and the results indicated that the resistance to VCR, DNR and L-Asp was increased in the Ik6 transfectants. The $\mathrm{IC}_{50}$ values of VCR (34.94 vs. $\left.20.51 \mathrm{ng} / \mathrm{ml}\right)$, DNR (12.25 vs. $1.89 \mathrm{ng} / \mathrm{ml}$ ) and L-Asp (2.37 vs. 0.36I U/ml) were higher than that of the control $(\mathrm{P}<0.05)$ (Fig. 5). The results from flow cytometry and western blotting revealed that Ik6 decreased the drug-induced apoptosis together with the upregulation of the bcl-xl protein (Fig. 6A and B).

Ik6 does not affect the invasiveness of Nalm-6 cells. Realtime PCR was performed to measure the expression of genes regulating invasion, and the results revealed no significant differences in the expression levels for the mRNA coding of 

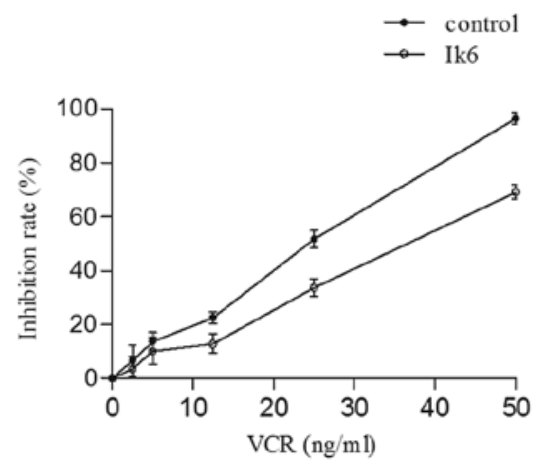

- control

$\rightarrow$ Ik6

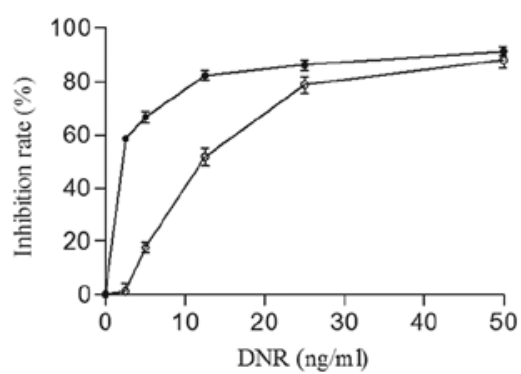

$\rightarrow$ control

$\rightarrow$ Ik6

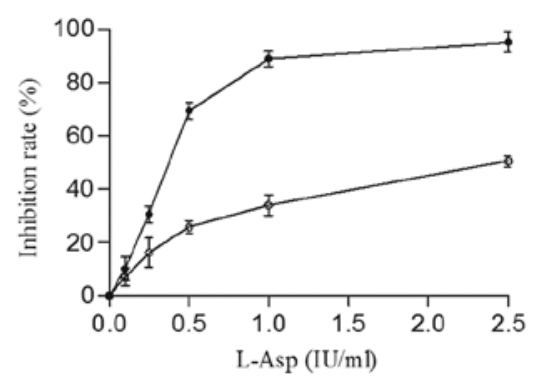

Figure 5. Ik6 overexpression enhances the chemoresistance of Nalm-6 cells. The CCK-8 assay revealed a dose-dependent growth inhibition, and the growth inhibition rate of Nalm-6/Ik6 cells was lower than that of the control cells following treatment with the same concentrations of VCR, DNR or L-Asp (P<0.05, $\mathrm{n}=5$ ). Ik6, Ikaros isoform 6; VCR, vincristine; DNR, daunorubicin; L-Asp, L-asparaginase.

A

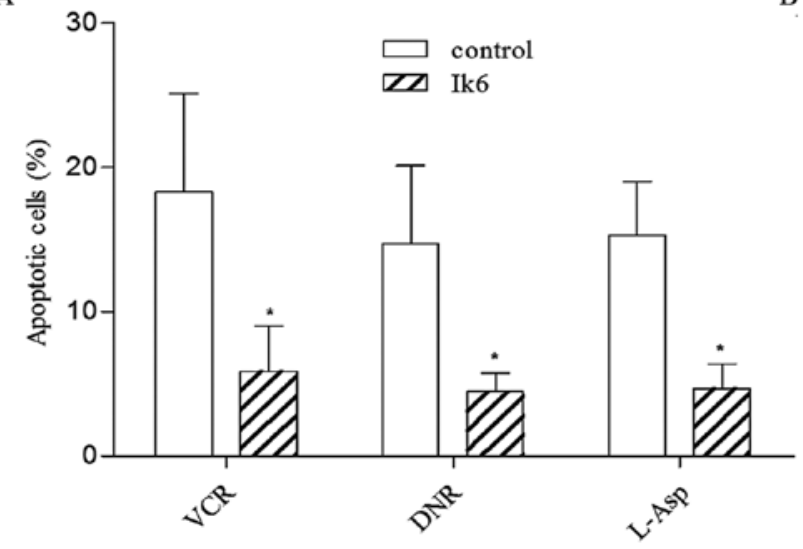

B

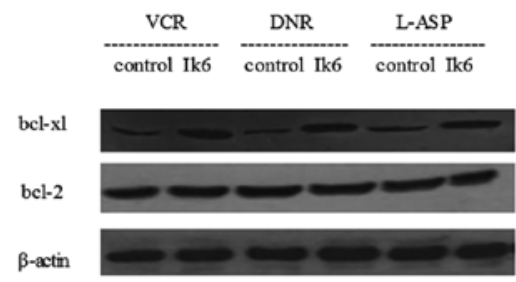

阝

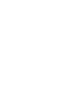

Figure 6. Ik6 overexpression decreases the drug-induced apoptosis of Nalm-6 cells. (A) The result of the flow cytometry revealed that, in contrast to the control cells, cell apoptosis induced by the drugs was markedly reduced in Nalm-6/Ik6 cells ( $\mathrm{P}<0.05, \mathrm{n}=3$ ). (B) Western blot analysis of apoptosis-related proteins showed that the expression of bcl-xl in Nalm-6/Ik6 cells was upregulated. Ik6, Ikaros isoform 6.

A

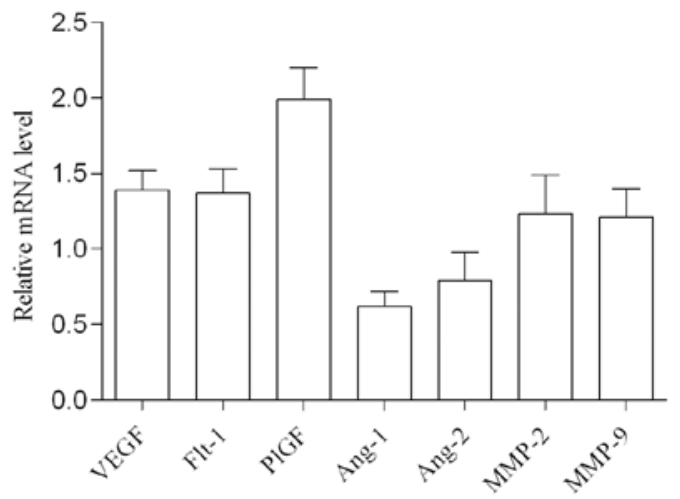

B

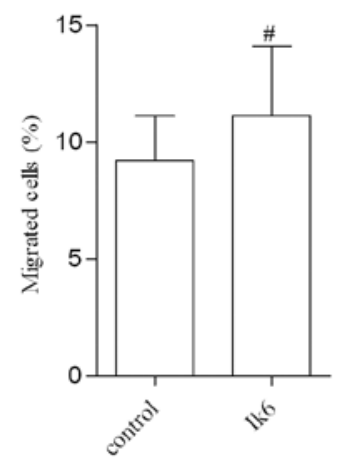

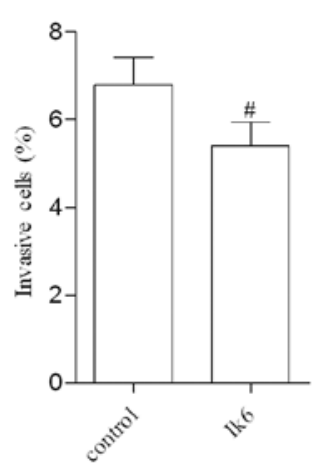

Figure 7. Ik6 overexpression has no effect on the invasive ability of leukemia cells. (A) The mRNA level of genes regulating invasion, VEGF, Flt-1, PlGF, Ang-1, Ang-2, MMP-2 and MMP-9, in Nalm-6/Ik6 cells was detected by real-time RT-PCR. Using Nalm-6/mock cells as the control, the result showed that there was no differences in the expression of these genes $(n=5)$. (B) The migration and invasion assays showed that there was no difference in migratory and invasive abilities between the Nalm-6/Ik6 cells and control cells $\left({ }^{*} \mathrm{P}>0.05, \mathrm{n}=3\right)$. Ik6, Ikaros isoform 6; VEGF, vascular endothelial growth factor; Flt-1, vascular endothelial growth factor receptor; PIGF, placenta growth factor fragment; Ang-1, angiogenin-1; Ang-2, angiogenin-2; MMP-2, matrix metalloproteinase-2; MMP-9, matrix metalloproteinase-9.

VEGF, Flt-1, PlGF, Ang1, Ang2, MMP-2 and MMP-9 between Nalm-6/Ik6 cells and the control (P>0.05) (Fig. 7A). In the migration and invasion assays, cells from both groups transmi- grated from the upper to the lower chamber, but the quantity of cells in the lower chamber was not statistically different $(\mathrm{P}>0.05)$ (Fig. 7B). 


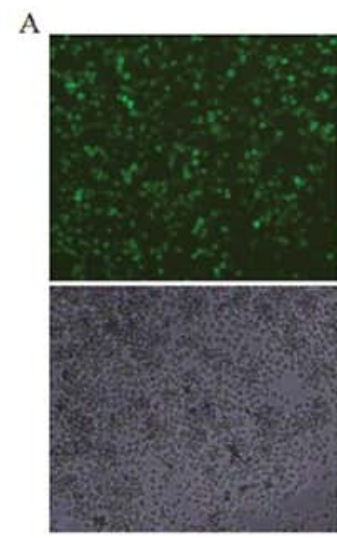

control

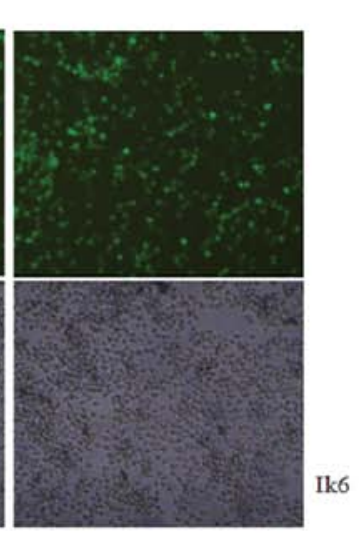

Ik6 shRNA

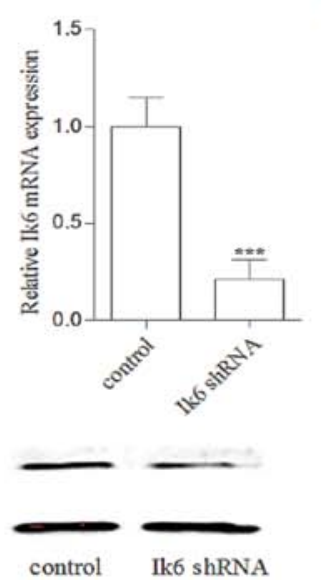

B

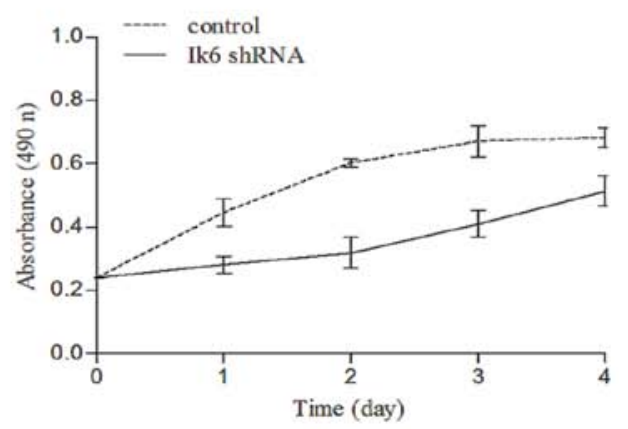

Figure 8. Silencing of Ik6 decreases the proliferation of Sup-B15 cells. (A) Lentiviral-mediated shRNA targeting Ik6 in Sup-B15 cells. The expression of Ik6 mRNA and protein was detected by real-time RT-PCR and western blotting, respectively. ${ }^{* * *} \mathrm{P}<0.001$, $\mathrm{n}=5$ ). (B) Silencing of Ik6 in Sup-B15 cells inhibited cell proliferation $\left({ }^{*} \mathrm{P}<0.05, \mathrm{n}=5\right)$. Ik6, Ikaros isoform 6; shRNA, small hairpin RNA.
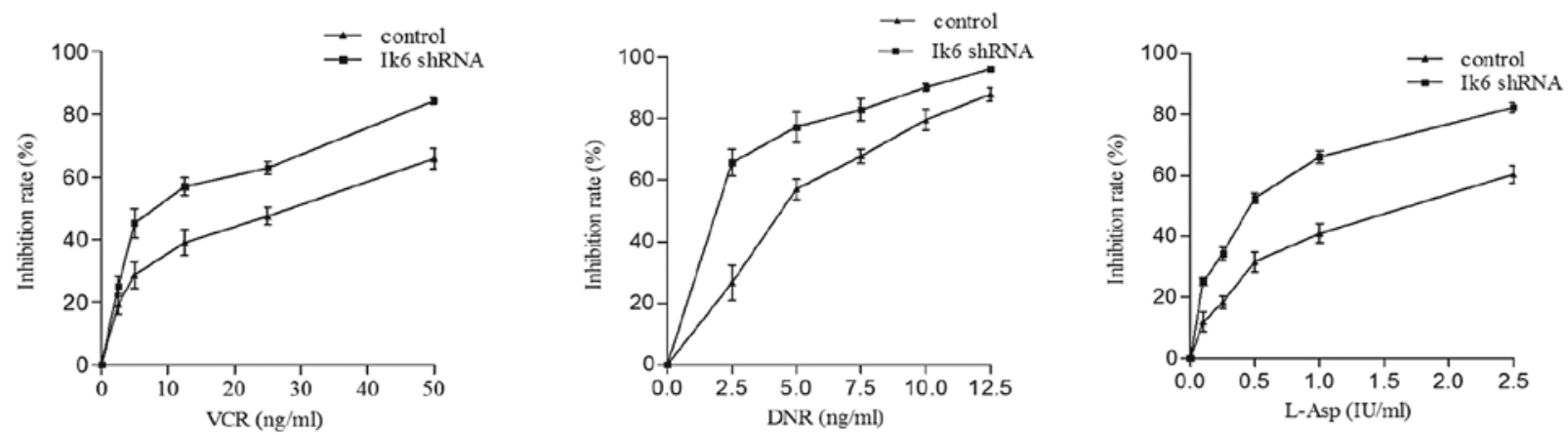

Figure 9. Silencing of Ik6 enhances the chemosensitivity of Sup-B15 cells to VCR, DNR and L-Asp (P<0.05, n=5). Ik6, Ikaros isoform 6; VCR, vincristine; DNR, daunorubicin; L-Asp, L-asparaginase.

Silencing of Ik6 in Sup-B15 cells inhibits proliferation and increases chemosensitivity. To further confirm the effect of Ik6 on proliferation and chemosensitivity of leukemia cells, Sup-B15 cells were modified to block Ik6 expression via a lentiviral-mediated shRNA vector. As shown in Fig. 8A, Ik6 mRNA and protein expression was downregulated in the Sup-B15/ Ik6 shRNA cells when compared with the control. Ik6 shRNA significantly inhibited the proliferative activity of Sup-B15 cells (Fig. 8B, $\mathrm{P}<0.05$ ) and enhanced the chemosensitivity to VCR, DNR and L-Asp. The $\mathrm{IC}_{50}$ values of control cells to VCR, DNR and L-Asp were 2.6-, 2.9- and 3.4-fold higher than these values in the Sup-B15/Ik6 shRNA cells $(\mathrm{P}<0.05$, Fig. 9).

\section{Discussion}

In an effort to understand the phenomenon of leukemia relapse, several predictors of the ultimate outcome have been identified in the hopes of providing clues that may lead to more effective treatment $(17,18)$. The Ik6 variant of the IKZF1 gene, an unfavorable prognostic marker in the outcome analysis of ALL, was independently associated with both overall survival and relapse-free survival (16). Ph/BCR-ABL was also known as a high-risk prognostic factor, but the emergence of tyrosine kinase inhibitors has significantly improved complete remission rates and the outcome of Ph-positive ALL patients $(19,20)$.
Therefore, just as $\mathrm{Ph}$ not only indicates risk but is also a therapeutic target, Ik6 may not only provide insight into leukemogenesis but may also lead to the establishment of new treatment strategies targeting ALL.

We previously reported that Ik6 expression in the bone marrow cells of newly diagnosed ALL patients is associated with a higher level of 33-day minimal residual disease, which indicated excessive proliferation and primary chemoresistance of leukemia cells (16). However, there have been few published data concerning the dynamic expression of Ik6 during chemotherapy. The present study demonstrated that Ik6 expression is downregulated by chemotherapeutic agents in vivo and in vitro. A high level of Ik6 mRNA expression was detected in relapsed patients. Thus, Ik6 is not only a predictor of poor prognosis at initial diagnosis but is also a marker for monitoring chemotherapeutic efficacy and relapse during treatment. Certainly, more data from Ik6-positive patients are needed to provide the relationship between the exact level of Ik6 mRNA expression and response to treatment and relapse.

To explore the potential role of Ik6 in the treatment of ALL, we evaluated the effect of Ik6 on leukemia cell growth and found that overexpression of Ik6 increased cell proliferation. The results were in accordance with these of studies on in vitro systems, which demonstrated that Ik6 transfection stimulated the proliferation of pituitary cells (21) and human $\mathrm{CD} 34^{+}$cord 
blood cells (22). Furthermore, Ik6-expressing cells progressed more rapidly through the cell cycle than the control cells, in as much as they peaked in the $\mathrm{S}$ phase earlier.

Additionally, we analyzed the role of Ik6 in leukemia cell chemosensitivity. Our study demonstrated that the overexpression of Ik6 increased the chemoresistance of leukemia cells in vitro. The Ik6-expressing Nalm-6 cells were 1.7 times more resistant to VCR, 6.5 times more resistant to DNR and 6.6 times more resistant to L-Asp. The following clinical studies support the above-mentioned results. Tonnelle et al (14) reported that the response of patients with positive expression of Ik6 to induction treatment was not favorable; 7 of 8 patients did not reach complete remission and 1 achieved remission at the end of the induction therapy. A large sample of clinical data showed that $16.07 \%$ of the patients with Ik6 did not achieve remission and $48.44 \%$ suffered from relapse (16). VCR, DNR and L-Asp are currently the first-line chemotherapeutic drugs for the treatment of pediatric ALL. All of these drugs can induce apoptosis of leukemic cells. In the present study, we found that when Ik6 expression was increased in Nalm-6 cells, following treatment with the three drugs, significantly enhanced proliferative activity of Nalm- 6 cells and a decreased level of apoptosis were noted with upregulation of bcl-xl. To further confirm the role of Ik6 in therapy, we found that silencing of Ik6 significantly inhibited proliferation and sensitizes Sup-B15 cells to the chemotherapeutic agents.

As well as acquired drug resistance, extramedullary tissue infiltration of leukemic cells is a major obstacle to leukemia treatment (23). Excessive egress of leukemia cell blasts results in invasion into various organs or tissues, such as the central nervous system (CNS) and testis $(24,25)$. The results of our studies on leukemia cell invasion indicated that there was no effect of Ik6 on the invasive ability of leukemia cells in vitro.

In the present study, we found that Ik6 may be utilized as a gene marker to predict the clinical efficacy of chemotherapy. The patients with Ik6 overexpression should receive more intensive therapy, and detection for multidrug resistance should be carried out. More importantly, Ik6 regulates the proliferation and chemosensitivity of leukemia cells, and anti-apoptosis may be the mechanism of action. Regulatory apoptosis pathways that are associated with Ik6 are a potential target for a novel strategy for the chemotherapy of ALL.

\section{Acknowledgements}

The authors would like to thank Dengli Hong and Zhen Li of the Department of Medical Stem Cell Biology of Shanghai Jiaotong University for supporting the present study. The study was supported by a grant from the National Nature Science Foundation of China (81300414).

\section{References}

1. Pui CH, Robison LL and Look AT: Acute lymphoblastic leukaemia. Lancet 371: 1030-1043, 2008.

2. Rivera GK, Zhou Y, Hancock ML, et al: Bone marrow recurrence after initial intensive treatment for childhood acute lymphoblastic leukemia. Cancer 103: 368-376, 2005.
3. Fielding AK, Richards SM, Chopra R, et al: Outcome of 609 adults after relapse of acute lymphoblastic leukemia (ALL); an MRC UKALL12/ECOG 2993 study. Blood 109: 944-950, 2007.

4. Thomas X, Boiron JM, Huguet F, et al: Outcome of treatment in adults with acute lymphoblastic leukemia: analysis of the LALA-94 trial. J Clin Oncol 22: 4075-4086, 2004.

5. Marjerrison S, Antillon F, Fu L, et al: Outcome of children treated for relapsed acute lymphoblastic leukemia in Central America. Cancer 119: 1277-1283, 2013.

6. Oriol A, Vives S, Hernández-Rivas JM, et al: Outcome after relapse of acute lymphoblastic leukemia in adult patients included in four consecutive risk-adapted trials by the PETHEMA Study Group. Haematologica 95: 589-596, 2010.

7. Wang JH, Nichogiannopoulou A, Wu L, et al: Selective defects in the development of the fetal and adult lymphoid system in mice with an Ikaros null mutation. Immunity 5: 537-549, 1996.

8. Winandy S, Wu P and Georgopoulos K: A dominant mutation in the Ikaros gene leads to rapid development of leukemia and lymphoma. Cell 83: 289-299, 1995.

9. Rebollo A and Schmitt C: Ikaros, Aiolos and Helios: transcription regulators and lymphoid malignancies. Immunol Cell Biol 81: 171-175, 2003.

10. Mullighan CG, Miller CB, Radtke I, et al: $B C R-A B L 1$ lymphoblastic leukaemia is characterized by the deletion of Ikaros. Nature 453: 110-114, 2008.

11. Iacobucci I, Lonetti A, Cilloni D, et al: Identification of different Ikaros cDNA transcripts in Philadelphia-positive adult acute lymphoblastic leukemia by a high-throughput capillary electrophoresis sizing method. Haematologica 93: 1814-1821, 2008.

12. Koipally J and Georgopoulos K: A molecular dissection of the repression circuitry of Ikaros. J Biol Chem 277: 27697-27705, 2002.

13. Mullighan CG, Su X, Zhang J, et al: Deletion of IKZFI and prognosis in acute lymphoblastic leukemia. N Engl J Med 360: 470-480, 2009.

14. Tonnelle C, Imbert MC, Sainty D, Granjeaud S, N'Guyen C and Chabannon C: Overexpression of dominant-negative Ikaros 6 protein is restricted to a subset of $\mathrm{B}$ common adult acute lymphoblastic leukemias that express high levels of the CD34 antigen. Hematol J 4: 104-109, 2003.

15. Zhou F, Mei H, Jin R, Li X and Chen X: Expression of Ikaros isoform 6 in Chinese children with acute lymphoblastic leukemia. J Pediatr Hematol Oncol 33: 429-432, 2011.

16. Mi JQ, Wang X, Yao Y, et al: Newly diagnosed acute lymphoblastic leukemia in China (II): prognosis related to genetic abnormalities in a series of 1091 cases. Leukemia 26: 1507-1516, 2012.

17. Harrison CJ: Cytogenetics of paediatric and adolescent acute lymphoblastic leukaemia. Br J Haematol 144: 147-156, 2009.

18. Szczepański T, Harrison CJ and van Dongen JJ: Genetic aberrations in paediatric acute leukaemias and implications for management of patients. Lancet Oncol 11: 880-889, 2010.

19. Mizuta S, Matsuo K, Yagasaki F, et al: Pre-transplant imatinibbased therapy improves the outcome of allogeneic hematopoietic stem cell transplantation for $B C R-A B L$-positive acute lymphoblastic leukemia. Leukemia 25: 41-47, 2011.

20. Wassmann B, Pfeifer H, Goekbuget N, et al: Alternating versus concurrent schedules of imatinib and chemotherapy as front-line therapy for Philadelphia-positive acute lymphoblastic leukemia ( $\mathrm{Ph}^{+}$ALL). Blood 108: 1469-1477, 2006.

21. Ezzat S, Zhu X, Loeper S, Fischer S and Asa SL: Tumor-derived Ikaros 6 acetylates the Bcl-XL promoter to up-regulate a survival signal in pituitary cells. Mol Endocrinol 20: 2976-2986, 2006.

22. Suzuki K, Ono R, Ohishi K, et al: IKAROS isoform 6 enhances BCR-ABL1-mediated proliferation of human CD $34^{+}$hematopoietic cells on stromal cells. Int J Oncol 40: 53-62, 2012.

23. Song JH, Kim SH, Cho D, Lee IK, Kim HJ and Kim TS: Enhanced invasiveness of drug-resistant acute myeloid leukemia cells through increased expression of matrix metalloproteinase- 2 . Int J Cancer 125: 1074-1081, 2009.

24. Suminoe A, Matsuzaki A, Hattori H, Koga Y, Ishii E and HaravT: Expression of matrix metalloproteinase (MMP) and tissue inhibitor of MMP (TIMP) genes in blasts of infant acute lymphoblastic leukemia with organ involvement. Leuk Res 31: 1437-1440, 2007.

25. Kaddu S, Zenahlik P, Beham-Schmid C, Kerl H and Cerroni L: Specific cutaneous infiltrates in patients with myelogenous leukemia: a clinicopathologic study of 26 patients with assessment of diagnostic criteria. J Am Acad Dermatol 40: 966-978, 1999. 